\title{
A Multi-Criteria Decision-Making Method Using Power Aggregation Operators for Single-valued Neutrosophic Sets
}

\author{
Lihua Yang and Baolin Li* \\ School of Economics and Management, Hubei University of Automotive \\ Technology, Shiyan, 442002, China \\ yanglh168@126.com;libaolin108@163.com
}

\begin{abstract}
As a generalization of intuitionistic fuzzy sets, neutrosophic sets (NSs) can be better handle the incomplete, indeterminate and inconsistent information, which have attracted the widespread concerns for researchers. In this paper, some new aggregation operators are proposed under single-valued neutrosophic environment. Firstly, the definition and operational laws of single-valued neutrosophic numbers (SVNNs) are introduced. Then, the single-valued neutrosophic power average (SVNPA) operator and the single-valued neutrosophic power weighted average (SVNPWA) operator are developed, and some properties of SVNPWA operator are also analyzed. Furthermore, a method for solving multi-criteria decision-making (MCDM) problems is explored based on the power aggregation operators and cosine similarity measures. Finally, an illustrative example is shown to verify the effectiveness and practicality of the proposed method.
\end{abstract}

Keywords: Multi-criteria decision-making, power aggregation operators, singlevalued neutrosophic sets

\section{Introduction}

In the real world, the decision-making problems with incomplete or inaccurate information are difficult to be precisely expressed by decision-makers. Under these circumstances, Zadeh [1] firstly proposed the theory of fuzzy sets (FSs), where the membership degree is presented using a crisp value between zero and one, have been applied successfully in many different fields. However, FSs only have a membership and lack non-membership degree. In order to solve the problem, Atanassov [1] proposed the intuitionistic fuzzy sets (IFSs), which is an extension of Zadeh's FSs. IFSs have been widely extended and got more attention in solving MCDM problems [3].

Although the theories of FSs and IFSs have been generalized, it can not handle all kinds of uncertainties in many cases. The indeterminate information and inconsistent information existing commonly in the real world can not be deal with by FSs and IFSs. For example, during a voting process, forty percent vote "yes", thirty percent vote "no", twenty percent are not sure, and ten percent give up. This issue is beyond the scope of IFSs, which cannot distinguish the information between unsure and giving up. Therefore, on the basis of IFSs, Smarandache [9] introduced neutrosophic logic and neutrosophic sets (NSs) by adding an independent indeterminacy-membership. Then, the aforementioned example can be expressed as $x(0.4,0.2,0.3)$ with

respect to NSs. Moreover, true-membership, indeterminacy-membership and falsemembership in NSs are completely independent, whereas the uncertainty is dependent on the true-membership and false-membership in IFSs. So the notion of NSs is more general and overcomes the aforementioned issues.

From scientific or engineering point of view, the neutrosophic set and set-theoretic operators will be difficult to apply in the real application without specific description. 
Therefore, a single-valued netrosophic set (SVNS) is proposed [8], which is an extension of NSs, and some properties of SVNS are also provided. Ye [9] proposed the correlation coefficient and weighted correlation coefficient of SVNSs, and proved that the cosine similarity degree is a special case of the correlation coefficient in SVNS. Majumdar [11]defined similarity measures between two SVNSs and introduced a measure of entropy of SVNSs. Ye [12] proposed the cross-entropy of SVNSs. Furthermore, Ye [13] introduced the concept of simplified neutrosophic sets (SNSs), and proposed a MCDM method using a simplified neutrosophic weighted arithmetic average operator and a simplified neutrosophic weighted geometric average operator. Liu [14] proposed a multiple attribute decision-making method based on single-valued neutrosophic normalized weighted Bonferroni mean.

Wang [15] proposed the concept of interval neutrosophic set (INS) and gave the settheoretic operators of INS. Zhang [16] defined the operations for INSs, and developed two interval neutrosophic number aggregation operators. Ye [17]defined the Hamming and Euclidean distances between INSs, and proposed the similarity measures between INSs based on the relationship between similarity measures and distances. Liu[18] proposed some Hamacher aggregation operators for the interval-valued intuitionistic fuzzy numbers. Peng [19] defined multi-valued NSs, and discussed operations based on Einstein. Liu [20] proposed the concept of the interval neutrosophic hesitant fuzzy set, presented the operations and developed generalized hybrid weighted aggregation operators.

Information aggregation is very important in MCDM and MCGDM problems, so various aggregation operators have been proposed and developed in the past years. Yager [21] and $\mathrm{Xu}$ [22] proposed weighted arithmetic average operator and weighted geometric average operator, which are two of the most common operators. Zhao [23] developed generalized aggregation operators for intuitionistic fuzzy sets (IFSs). The NSs is an extension of IFSs, so it is significant meaningful to research the aggregation operators for NSs. However, until to now, there are a few researches on aggregation operators for SVNSs, and apply them to decision-making problems. Many traditional aggregation operators do not consider the relationship of different input arguments in the decision process. Yager [24] firstly defined the power average operator which makes the arguments support each other. $\mathrm{Xu}$ [25] introduced the power geometric operator. Zhou [26] developed a generalized power average operator. Liu [27] defined intuitionistic trapezoidal fuzzy power generalized aggregation operator. However, power average operators have not been applied to handle MCDM problems under single-valued neutrosophic environment. Therefore, the aim of the paper is to develop single-valued neutrosophic power average aggregation operators. Meanwhile, we will discuss its properties, such as idempotency, commutativity.

The rest of paper is organized as follows. In Section 2, we introduce some concepts and operations of SVNS. New power aggregation operators for SVNN are defined, and some properties are discussed in Section 3. Section 4 establishes the detail decision method for multi-criteria decision making based on the proposed operators under single-valued neutrosophic fuzzy information environment. Section 5 presents an illustrative example according to our method. Finally, the main conclusions of this paper are summarized in Section 6.

\section{Preliminaries}

In this Section, some concepts and definitions with respect to SVNs are introduced, which will be utilized in the remainder of the paper.

Definition 1 [8]. Let $X$ be a universe of discourse, with a generic element in $X$ denoted by $x$. A NS $A$ in $X$ is

$$
A=\left\{\left\langle x, T_{A}(x), I_{A}(x), F_{A}(x)\right\rangle \mid x \in X\right\},
$$


Where $T_{A}(x)$ is the truth-membership function, $I_{A}(x)$ is the indeterminacymembership function, and $F_{A}(x)$ is the falsity-membership function. $T_{A}(x), I_{A}(x)$ and $F_{A}(x)$ are real standard or nonstandard subsets of $] 0^{-}, 1^{+}[$. There is no restriction on the sum of $T_{A}(x), I_{A}(x)$ and $F_{A}(x)$, so $0^{-} \leq \sup T_{A}(x)+\sup I_{A}(x)+\sup F_{A}(x) \leq 3^{+}$.

Definition 2 [9]. Let $X$ be a universe of discourse, with a generic element in $X$ denoted by $x$. A SVNS $A$ in $X$ is

$$
A=\left\{\left\langle x, T_{A}(x), I_{A}(x), F_{A}(x)\right\rangle \mid x \in X\right\},
$$

For each point $x$ in $X$,we have $T_{A}(x), I_{A}(x), F_{A}(x) \in[0,1]$, and $0 \leq T_{A}(x)+I_{A}(x)+F_{A}(x) \leq 3$. A SVNS is an instance of a NS, and simplified neutrosophic set (SNS) is a subclass of NS, so SVNS is also an special case of SNS. For convenience, we can use $x=\left(T_{x}, I_{x}, F_{x}\right)$ to represent an element in SVNS, and call it a single-valued neutrosophic number (SVNN). The set of all SVNNs is represented as SVNNS.

Definition 3[13].Let $x=\left(T_{1}, I_{1}, F_{1}\right)$ and $y=\left(T_{2}, I_{2}, F_{2}\right)$ be two SVNNs, then operational relations are defined as follows:

(1) $x \oplus y=\left(T_{1}+T_{2}-T_{1} T_{2}, I_{1}+I_{2}-I_{1} I_{2}, F_{1}+F_{2}-F_{1} F_{2}\right)$

(2) $x \otimes y=\left(T_{1} T_{2}, I_{1} I_{2}, F_{1} F_{2}\right)$

(3) $\lambda x=\left(1-\left(1-T_{1}\right)^{\lambda}, 1-\left(1-I_{1}\right)^{\lambda}, 1-\left(1-F_{1}\right)^{\lambda}\right) \quad \lambda>0$

(4) $x^{\lambda}=\left(T_{1}^{\lambda}, I_{1}^{\lambda}, F_{1}^{\lambda}\right) \quad \lambda>0$

There are some limitations related to definition 3[16], and some novel operations are defined.

Definition 4. Let $x=\left(T_{1}, I_{1}, F_{1}\right)$ and $y=\left(T_{2}, I_{2}, F_{2}\right)$ be two SVNNs, then operational relations are defined as follows:

(1) $x \oplus y=\left(T_{1}+T_{2}-T_{1} T_{2}, I_{1} I_{2}, F_{1} F_{2}\right)$

(2) $x \otimes y=\left(T_{1} T_{2}, I_{1}+I_{2}-I_{1} I_{2}, F_{1}+F_{2}-F_{1} F_{2}\right)$

(3) $\lambda x=\left(1-\left(1-T_{1}\right)^{\lambda}, I_{1}^{\lambda}, F_{1}^{\lambda}\right) \quad \lambda>0$

(4) $x^{\lambda}=\left(T_{1}^{\lambda}, 1-\left(1-I_{1}\right)^{\lambda}, 1-\left(1-F_{1}\right)^{\lambda}\right) \quad \lambda>0$

Definition 5. Let $x=\left(T_{1}, I_{1}, F_{1}\right)$ and $y=\left(T_{2}, I_{2}, F_{2}\right)$ be any two SVNNs, then the Hamming distance between $x$ and $y$ can be defined as follows:

$$
d(x, y)=\left|T_{1}-T_{2}\right|+\left|I_{1}-I_{2}\right|+\left|F_{1}-F_{2}\right|
$$

Definition 6. Let $x=(T, I, F)$ be a SVNN, and the cosine similarity measure $S(x)$ between SVNN $x$ and the ideal alternative $(1,0,0)$ can be defined as follows:

$$
S(x)=\frac{T}{\sqrt{T^{2}+I^{2}+F^{2}}}
$$

\section{The Single-Valued Neutrosophic Power Aggregation Operators}

In this Section, the power aggregation operators of SVNNs are defined, and some properties are discussed. 


\subsection{Power Average Operator}

Yager[24] introduced the power average(PA) operator, which is nonlinear weighted average aggregation operator.

Definition 7.The PA operator is the mapping defined as

$$
P A\left(a_{1}, \cdots, a_{n}\right)=\frac{\sum_{i=1}^{n}\left(1+T\left(a_{i}\right)\right) a_{i}}{\sum_{j=1}^{n}\left(1+T\left(a_{j}\right)\right)}
$$

Where $T\left(a_{i}\right)=\sum_{j=1, j \neq i}^{n} \operatorname{Supp}\left(a_{i}, a_{j}\right)$, and $\operatorname{Supp}\left(a_{i}, a_{j}\right)$ is the support for $a_{i}$ and $a_{j}$. The properties are as follows:

(1) $\operatorname{Supp}\left(a_{i}, a_{j}\right) \in[0,1]$

(2) $\operatorname{Supp}\left(a_{i}, a_{j}\right)=\operatorname{Supp}\left(a_{j}, a_{i}\right)$

(3) $\operatorname{Supp}\left(a_{i}, a_{j}\right) \geq \operatorname{Supp}\left(a_{p}, a_{q}\right) \operatorname{iffd}\left(a_{i}, a_{j}\right)<d\left(a_{p}, a_{q}\right)$

Where $d\left(a_{i}, a_{j}\right)$ is the distance between $a_{i}$ and $a_{j}$. The smaller distance is, the more they support each other.

\subsection{Power Weighted Average Operator}

Definition 8.Let $A_{j}=\left\langle T_{A_{j}}, I_{A_{j}}, F_{A_{j}}\right\rangle(j=1,2, \cdots, n)$ be a collection of SVNNs, and $w=\left(w_{1}, w_{2}, \cdots, w_{n}\right)$ be the weight vector of $A_{j}(j=1,2, \cdots, n)$, satisfying $w_{j} \geq 0$ and $\sum_{j=1}^{n} w_{j}=1$.The single-valued neutrosophic power weighted average (SVNPWA) operator of dimension $\mathrm{n}$ is the mapping SVNPWA: $S V N N^{n} \rightarrow S V N N$, then the SVNPWA operator is defined as

$$
\operatorname{SVNPWA}\left(A_{1}, A_{2}, \cdots, A_{n}\right)=\frac{\sum_{i=1}^{n} w_{i}\left(1+T\left(A_{i}\right)\right) A_{i}}{\sum_{j=1}^{n} w_{j}\left(1+T\left(A_{j}\right)\right)}
$$

Where $T\left(A_{i}\right)=\sum_{j=1, j \neq i}^{n} w_{j} \operatorname{Supp}\left(A_{i}, A_{j}\right)$, and $\operatorname{Supp}\left(A_{i}, A_{j}\right)$ is the support for $A_{i}$ and $A_{j}$, then the following conditions are true.

(1) $\operatorname{Supp}\left(A_{i}, A_{j}\right) \in[0,1]$

(2) $\operatorname{Supp}\left(A_{i}, A_{j}\right)=\operatorname{Supp}\left(A_{j}, A_{i}\right)$

(3) $\operatorname{Supp}\left(A_{i}, A_{j}\right) \geq \operatorname{Supp}\left(A_{p}, A_{q}\right) \operatorname{iffd}\left(A_{i}, A_{j}\right)<d\left(A_{p}, A_{q}\right)$

Where $\mathrm{d}$ is the distance measure defined in Definition 5.

Theorem1.For a SVNS $A_{j}(j=1,2, \cdots, n)$, we have the following result. 
$\operatorname{SVNPWA}\left(A_{1}, A_{2}, \cdots, A_{n}\right)$

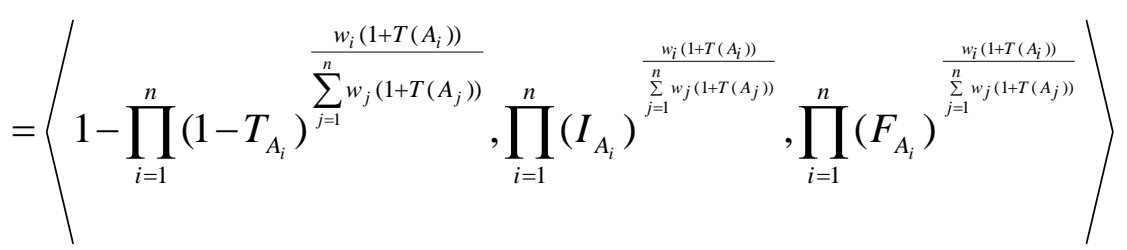

Proof. The proof can be done by using the mathematical induction. For simplicity, let $\xi_{i}=\frac{w_{i}\left(1+T\left(A_{i}\right)\right)}{\sum_{j=1}^{n} w_{j}\left(1+T\left(A_{j}\right)\right)}$ in the process of proof.

(1) when $\mathrm{n}=2$, then

$$
\begin{aligned}
& \xi_{1} A_{1}=<1-\left(1-T_{A_{1}}\right)^{\xi_{1}}, I_{A_{1}}^{\xi_{1}}, F_{A_{1}}^{\xi_{1}}> \\
& \xi_{2} A_{2}=<1-\left(1-T_{A_{2}}\right)^{\xi_{2}}, I_{A_{2}}^{\xi_{2}}, F_{A_{2}}^{\xi_{2}}>
\end{aligned}
$$

Thus,

$$
\begin{aligned}
& \operatorname{SVNPWA}\left(A_{1}, A_{2}\right)=\xi_{1} A_{1}+\xi_{2} A_{2} \\
& =<1-\left(1-T_{A_{1}}\right)^{\xi_{1}^{1}}+1-\left(1-T_{A_{2}}\right)^{\xi_{2}}-\left(1-\left(1-T_{A_{1}}\right)^{\xi_{1}}\right)\left(1-\left(1-T_{A_{2}}\right)^{\xi_{2}}\right), I_{A_{1}}^{\xi_{1}} I_{A_{2}}^{\xi_{2}}, F_{A_{1}}^{\xi_{1}} F_{A_{2}}^{\xi_{2}}> \\
& =<1-\left(1-T_{A_{1}}\right)^{\xi_{1}}\left(1-T_{A_{2}}\right)^{\xi_{2}}, I_{A_{1}} I_{A_{2}}, F_{A_{1}}^{\xi_{1}} F_{A_{2}}^{\xi_{2}}>
\end{aligned}
$$

(2) when $\mathrm{n}=\mathrm{k}$, by applying Equation (5), we get

$S V N P W A\left(A_{1}, A_{2}, \cdots, A_{k}\right)$

$$
=\left\langle 1-\prod_{i=1}^{k}\left(1-T_{A_{i}}\right)^{\xi_{i}}, \prod_{i=1}^{k}\left(I_{A_{i}}\right)^{\xi_{i}}, \prod_{i=1}^{k}\left(F_{A_{i}}\right)^{\xi_{i}}\right\rangle
$$

(3) when $\mathrm{n}=\mathrm{k}+1$, by applying Equation (6) and (7), we can get

$\operatorname{SVNPWA}\left(A_{1}, A_{2}, \cdots, A_{k}, A_{k+1}\right)$

$$
\begin{aligned}
&=\left\langle\begin{array}{l}
1-\prod_{i=1}^{k}\left(1-T_{A_{i}}\right)^{\xi_{i}}+1-\left(1-T_{A_{k+1}}\right)^{\xi_{k+1}}-\left(1-\prod_{i=1}^{k}\left(1-T_{A_{i}}\right)^{\xi_{i}}\right)\left(1-\left(1-T_{A_{k+1}}\right)^{\xi_{k+1}}\right), \\
\prod_{i=1}^{k}\left(I_{A_{i}}\right)^{\xi_{i}}\left(I_{A_{k+1}}\right)^{\xi_{k+1}}, \prod_{i=1}^{k}\left(F_{A_{i}}\right)^{\xi_{i}}\left(F_{A_{k+1}}\right)^{\xi_{k+1}}
\end{array}\right\rangle \\
&=\left\langle 1-\prod_{i=1}^{k+1}\left(1-T_{A_{i}}\right)^{\xi_{i}}, \prod_{i=1}^{k+1}\left(I_{A_{i}}\right)^{\xi_{i}}, \prod_{i=1}^{k+1}\left(F_{A_{i}}\right)^{\xi_{i}}\right\rangle
\end{aligned}
$$

Therefore, considering these results, we can get Equation (5) holds for any n. The proof is completed. Then by Theorem1, we know that the aggregated result using the SVNPWA operator is still a SVNN.

Theorem2. For a SVNS $A_{j}(j=1,2, \cdots, n)$, we have the following result.

$$
\begin{aligned}
& \operatorname{SVNPA}\left(A_{1}, A_{2}, \cdots, A_{n}\right)
\end{aligned}
$$

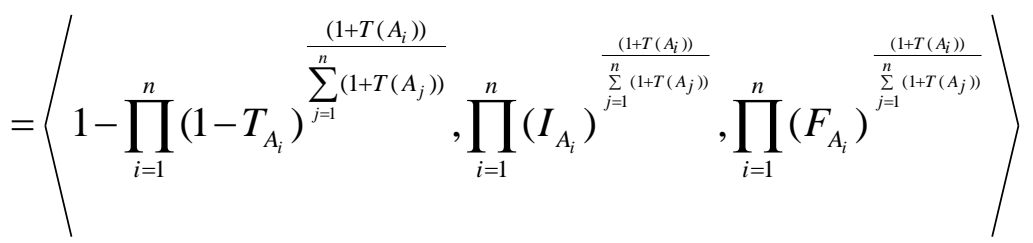

Then the aggregated result using the SVNPA operator is still a SVNN.

The SVNPWA operator has the following properties. 
(1) Idempotency: Let $A_{j}=\left\langle T_{A_{j}}, I_{A_{j}}, F_{A_{j}}\right\rangle(j=1,2, \cdots, n)$ be a collection of SVNNs, and $A=\left\langle T_{A}, I_{A}, F_{A}\right\rangle$ be a SVNN. If $A_{j}=A \quad(j=1,2, \cdots, n)$, then $\operatorname{SVNPWA}\left(A_{1}, A_{2}, \cdots, A_{n}\right)=A$.

(2) Commutativity: Let $A_{j}=\left\langle T_{A_{j}}, I_{A_{j}}, F_{A_{j}}\right\rangle(j=1,2, \cdots, n)$ be a collection of SVNNs, if $A_{j}^{*}(j=1,2, \cdots, n)$ is any permutation of $A_{j}(j=1,2, \cdots, n)$, then $\operatorname{SVNPWA}\left(A_{1}, A_{2}, \cdots, A_{n}\right)=\operatorname{SVNPWA}\left(A_{1}^{*}, A_{2}^{*}, \cdots, A_{n}^{*}\right)$.

Boundedness:

Let $A_{j}^{-}=\left\langle T_{A_{j}^{-}}, I_{A_{j}^{-}}, F_{A_{j}^{-}}\right\rangle(j=1,2, \cdots, n), A_{j}=\left\langle T_{A_{j}}, I_{A_{j}}, F_{A_{j}}\right\rangle(j=1,2, \cdots, n), \mathrm{a}$ nd $A_{j}^{+}=\left\langle T_{A_{j}^{+}}, I_{A_{j}^{+}}, F_{A_{j}^{+}}\right\rangle(j=1,2, \cdots, n)$ be three collections of SVNNs. If for all j, $T_{A_{j}^{-}} \leq T_{A_{j}} \leq T_{A_{j}^{+}}, I_{A_{j}^{+}} \leq I_{A_{j}} \leq I_{A_{j^{-}}}$, and $F_{A_{j}} \leq F_{A_{j}} \leq F_{A_{j^{-}}}$, then $\operatorname{SVNPWA}\left({A_{1}^{-}}^{-},{A_{2}}^{-}, \cdots,{A_{n}^{-}}^{-}\right) \leq \operatorname{SVNPWA}\left(A_{1}, A_{2}, \cdots, A_{n}\right) \leq \operatorname{SVNPWA}\left({A_{1}^{+}}^{+},{A_{2}^{+}}^{+}, \cdots,{A_{n}^{+}}^{+}\right)$

\section{The Multi-Criteria Decision-Making Method Based on the SVNPWA Operator}

In this Section, we will use the SVNPWA operator to deal with the multi-criteria decision-making problems under the single-valued neutrosophic environment, where the alternative values are in the form of SVNNs and the criteria weights are in the form of crisp values.

For a multiple criteria decision making problem, suppose there are $m$ alternatives denoted by $A=\left\{A_{1}, A_{2}, \cdots, A_{m}\right\}$ and $n$ criteria denoted by $C=\left\{C_{1}, C_{2}, \cdots, C_{n}\right\}$, and the corresponding weights of criteria given by decision-maker are $w=\left\{w_{1}, w_{2}, \cdots, w_{n}\right\}$, where $w_{j} \geq 0(j=1,2, \cdots, n)$, and $\sum_{j=1}^{n} w_{j}=1$. The evaluation value of $A_{i} \quad(i=1,2, \cdots, m)$ for criteria $C_{j}(j=1,2, \cdots, n)$ entered by decision-maker is represented in the form of SVNS.

$$
A_{i}=\left\{\left\langle C_{j}, T_{A_{i}}\left(C_{j}\right), I_{A_{i}}\left(C_{j}\right), F_{A_{i}}\left(C_{j}\right) \mid C_{j} \in C\right\rangle\right\}
$$

An SVNS is denoted by $\alpha_{i j}=\left\langle t_{i j}, i_{i j}, f_{i j}\right\rangle,(i=1,2, \cdots, m ; j=1,2, \cdots, n)$ for convenience. Therefore, the single-valued neutrosophic decision matrix can be found as follows:

$$
D=\left(\alpha_{i j}\right)_{m \times n}=\left[\begin{array}{cccc}
\left\langle t_{11}, i_{11}, f_{11}\right\rangle & \left\langle t_{12}, i_{12}, f_{12}\right\rangle & \cdots & \left\langle t_{1 n}, i_{1 n}, f_{1 n}\right\rangle \\
\left\langle t_{21}, i_{21}, f_{21}\right\rangle & \left\langle t_{22}, i_{22}, f_{22}\right\rangle & \cdots & \left\langle t_{2 n}, i_{2 n}, f_{2 n}\right\rangle \\
\vdots & \vdots & \vdots & \vdots \\
\left\langle t_{m 1}, i_{m 1}, f_{m 1}\right\rangle & \left\langle t_{m 2}, i_{m 2}, f_{m 2}\right\rangle & \cdots & \left\langle t_{m n}, i_{m n}, f_{m n}\right\rangle
\end{array}\right]
$$

Then, according to the SVNPWA operator, the aggregating single-valued netrosophic value of each alternative can be calculated by using Equation(5) for each row in the decision matrix $D$.

In multi-criteria decision-making problems, to obtain the ranking order of each alternative, we need to calculate the cosine similarity measure between an 
alternative $A_{i}$ and the ideal alternative $A^{*}$.Here, the $A^{*}$ is defined as $\alpha^{*}=\langle 1,0,0\rangle$,although the ideal alternative may not exist in real world.

$$
S_{i}\left(A_{i}, A^{*}\right)=\frac{t_{i} t^{*}+i_{i} i^{*}+f_{i} f^{*}}{\sqrt{t_{i}^{2}+i_{i}^{2}+f_{i}^{2}} \sqrt{\left(t_{i}^{*}\right)^{2}+i_{i}^{* 2}+f_{i}^{* 2}}}=\frac{t_{i}}{\sqrt{t_{i}^{2}+i_{i}^{2}+f_{i}^{2}}}
$$

The bigger the similarity measure value is, the better the alternative is. Then, the alternatives can be ordered and the best alternative can be identified.

Finally, we can have the following decision-making procedure for the MCDM problems.

Step1: Utilize the SVNPWA operator expressed by Equation(5) to calculate the comprehensive evaluation value of each alternative..

Step2: Utilize the cosine similarity measure expressed by Equation(2) to calculate the similarity of each alternative and the ideal alternative.

Step3: Give the ranking order of all alternatives based on the obtained measure values.

Step4: Get the best alternative and the worst alternative.

\section{Illustrative Example}

In this Section, an example of MCDM problems is presented in order to demonstrate the effectiveness and application of the mentioned decision-making method.

We will cite an example adapted from Ye [13]. There is an investment company, which wants to invest a sum of money in the best option. There is a panel with four possible alternatives for investing the money: (1) $A_{1}$ is a car company; (2) $A_{2}$ is a food company; (3) $A_{3}$ is a computer company; (4) $A_{4}$ is an arms company. The investment company must take a decision based on the following three criteria, which are denoted by $C_{j}(j=1,2,3):$ (1) $C_{1}$ is the risk analysis; (2) $C_{2}$ is the growth analysis; (3) $C_{3}$ is the environmental impact analysis. Then, the weight vector of the criteria is given by $W=(0.35,0.25,0.4)$.

The evaluation value of an alternative related to a criterion is obtained from the questionnaire of an expert. For example, when we ask the opinion of an expert about an alternative under the criterion, he or she may say that the possibility in which the statement is good is 0.3 , and the statement is poor is 0.5 , and the degree in which he or she is not sure is 0.2 . Then, it can be expressed as $\langle 0.3,0.2,0.5\rangle$ in the form of SVNNs. All of the possible values for an alternative with respect to a criteria are collected. Thus, forms:

the single-valued netrosophic decision matrix can be derived in the following

$$
D=\left[\begin{array}{lll}
\langle 0.4,0.2,0.3\rangle & \langle 0.4,0.2,0.3\rangle & \langle 0.2,0.2,0.5\rangle \\
\langle 0.6,0.1,0.2\rangle & \langle 0.6,0.1,0.2\rangle & \langle 0.5,0.2,0.2\rangle \\
\langle 0.3,0.2,0.3\rangle & \langle 0.5,0.2,0.3\rangle & \langle 0.5,0.3,0.2\rangle \\
\langle 0.7,0.0,0.1\rangle & \langle 0.6,0.1,0.2\rangle & \langle 0.4,0.3,0.2\rangle
\end{array}\right]
$$

To solve this problem, the computational steps are shown as follows: 
Step1: Calculate the evaluation value $\alpha_{i}$ for each alternative $A_{i}(i=1,2,3,4)$ by using the SVNPWA operator. For simplicity, suppose the weights of each criterion are equal, then the SVNPWA operator can be reduced to SVNPA operator.

Firstly, the support can be obtained by the following formula:

$\operatorname{Supp}\left(\alpha_{i j}, \alpha_{i k}\right)=1-d\left(\alpha_{i j}, \alpha_{i k}\right)$

Here, $i=1,2,3,4 ; j, k=1,2,3 ; j \neq k, \operatorname{Supp}\left(\alpha_{i j}, \alpha_{i k}\right)$ is the support, and satisfies the conditions shown in Definition 8. $d\left(\alpha_{i j}, \alpha_{i k}\right)$ is the Hamming distance between $\alpha_{i j}$ and $\alpha_{i k}$ given in Definition 5.

Then the following results can be given.

$$
\begin{aligned}
& T\left(\alpha_{11}\right)=1.6, T\left(\alpha_{12}\right)=1.6, T\left(\alpha_{13}\right)=1.2, \\
& T\left(\alpha_{21}\right)=1.8, T\left(\alpha_{22}\right)=1.8, T\left(\alpha_{23}\right)=1.6, \\
& T\left(\alpha_{31}\right)=1.4, T\left(\alpha_{32}\right)=1.6, T\left(\alpha_{33}\right)=1.4, \\
& T\left(\alpha_{41}\right)=1.0, T\left(\alpha_{42}\right)=1.3, T\left(\alpha_{43}\right)=0.9,
\end{aligned}
$$

Finally, we can get

$$
\begin{aligned}
& \alpha_{1}=<0.3464,0.2000,0.3492>, \\
& \alpha_{2}=<0.5707,0.1246,0.2000>, \\
& \alpha_{3}=<0.4423,0.2281,0.2630>, \\
& \alpha_{4}=<0.5872,0.0000,0.1599>,
\end{aligned}
$$

Step2: Calculate cosine similarity measure $S\left(\alpha_{i}, \alpha^{*}\right)(i=1,2,3,4)$ by using Equation (2) in Definition 2.

$$
\begin{aligned}
& S\left(\alpha_{1}, \alpha^{*}\right)=0.6524, S\left(\alpha_{2}, \alpha^{*}\right)=0.9243, \\
& S\left(\alpha_{3}, \alpha^{*}\right)=0.7858, S\left(\alpha_{4}, \alpha^{*}\right)=0.9649,
\end{aligned}
$$

Step3: Rank the alternatives.

We can get the ranking order of four alternatives on the basis of the similarity measure values. That is $A_{4} \succ A_{2} \succ A_{3} \succ A_{1}$

Step 4: The best alternative is $A_{4}$, and the worst alternative is $A_{1}$. This ranking result is the same as that is Ye [13], in which the weighted arithmetic average operator and the weighted geometric average operator are used.

\section{Conclusions}

In real situations, the information given by decision maker is often incomplete, inconsistent, and indeterminate. The single-valued neutrosophic set is an extension of traditional fuzzy set. Because SVNS can handle all above information, SVNS is more suitable for real scientific and engineering application. Power operator as an important aggregation operator can consider the relationship of different input arguments in the decision process. Therefore, in this paper, we proposed the SVNPA and SVNPWA operators under the single-valued neutrosophic environments. Moreover, some properties of the operator were provided in detail. Then, a MCDM method was established according to the proposed operators. Finally, an illustrative example was presented in order to demonstrate the effectiveness and application of 
the proposed method. The result shows that the multi-criteria decision-making method using power aggregation operators for single-valued neutrosophic sets is more practical.

In the future, we will study some new aggregation operators under neutrosophic environments, and give the application of the proposed operator to the other fields.

\section{Acknowledgement}

This paper is granted by Special Research Foundation of Young Teachers of Hubei University of Automotive Technology (No. 2012XQ02). The authors would like to thank the editors and the anonymous reviewers for their helpful and constructive comments.

\section{References}

[1] L. A. Zadeh, "Fuzzy sets", Information and Control, vol. 8, no. 3, (1965), pp. 338-353.

[2] K. T. Atanassov, "Intuitionistic fuzzy sets", Fuzzy Sets and Systems, vol. 20, no. 1, (1986), pp. 87-96.

[3] K. T. Atanassov, "Two theorems for intuitionistic fuzzy sets", Fuzzy Sets and Systems, vol. 110, no. 2, (2000), pp. 267-269.

[4] H. W. Liu and G. J. Wang, "Multi-criteria decision-making methods based on intuitionistic fuzzy sets", European Journal of Operational Research, vol. 179, no. 1, (2007), pp. 220-233.

[5] Z. Pei and L. Zheng, "A novel approach to multi-attribute decision making based on intuitionistic fuzzy sets", Expert Systems with Applications, vol. 39, no. 3, (2012),pp. 2560-2566.

[6] T. Y. Chen, "An outcome-oriented approach to multi-criteria decision analysis with intuitionistic fuzzyoptimistic/pessimistic operators", Expert Systems with Applications, vol. 37, no. 12, (2010), pp. 77627774.

[7] S. Zeng and W. Su, "Intuitionistic fuzzy ordered weighted distance operator", Knowledge-Based Systems, vol. 24, no. 8, (2011), pp. 1224-1232

[8] Z. S. Xu, "Intuitionistic fuzzy multi-attribute decision making: an interactive method", IEEE Transactions on Fuzzy Systems, vol.20, no. 3, (2012), pp. 514-525.

[9] F. Smarandache, "A unifying field in logics. neutrosophy: Neutrosophic probability, set and logic", American Research Press, Rehoboth, (1999).

[10] H. Wang, F. Smarandache, Y. Q. Zhang and R. Sunderraman, "Single valued neutrosophic sets", Multispace and Multi-structure, vol. 4, (2010), pp. 410-413

[11] J. Ye, "Multi-criteria decision-making method using the correlation coefficient under single-valued neutrosophic environment", International Journal of General Systems, vol. 42, no. 4, (2013), pp. 386394.

[12] P. Majumdar and S. K. Samanta, "On similarity and entropy of neutrosophic sets", Journal of Intelligent \& Fuzzy Systems, vol.26, no. 3, (2014), pp. 1245-1252.

[13] J. Ye, "Single valued neutrosophic cross-entropy for multi-criteria decision making problems", Applied Mathematical Modeling, vol.38, no. 3, (2014), pp. 1170-1175

[14] J. Ye, "A multi-criteria decision-making method using aggregation operators for simplified neutrosophic sets", Journal of Intelligent \& Fuzzy Systems, vol.26, no. 5, (2014), pp. 2459-2466.

[15] P. D. Liu and Y. M. Wang, "multiple attribute decision-making method based on single-valued neutrosophic normalized weighted Bonferroni mean", Neural Computing \& Application, vol. 25, (2014), pp. 2001-2010.

[16] H. Wang, F. Smarandache, Y. Q. Zhang and R. Sunderraman, "Interval Neutrosophic Sets and Logic: Theory and Applications in Computing", Hexis, Phoenix, Ariz, USA, (2005).

[17] H. Y. Zhang, J. Q. Wang and X. H. Chen, "Interval neutrosophic sets and their application in multicriteria decision making problems," The Scientific World Journal, vol. 2014, (2014).

[18] J. Ye, "Similarity measures between interval neutrosophic sets and their applications in multi-criteria decision-making", Journal of Intelligent and Fuzzy Systems, vol. 26, no. 1, (2014), pp. 165-172.

[19] P. D. Liu, "Some Hamacher aggregation operators based on the interval-valued intuitionistic fuzzy numbers and their application to Group Decision Making", IEEE Trans. on Fuzzy systems, vol. 22, no. 1, (2014), pp. 83-97.

[20] J. J. Peng, J. Q. Wang and X. H. Wu, "Multi-valued Neutrosophic Sets and Power Aggregation Operators with Their Application in Multi-criteria Group Decision-making Problems", International Journal of Computational Intelligence Systems, vol.8, no. 2, (2015), pp. 345-363.

[21] P. D. Liu and L. L Shi, "The generalized hybrid weighted average operator based on interval neutrosophic hesitant set and its application to multiple attribute decision making", Neural Computing \& Application, vol. 26, (2015), pp. 457-471.

[22] R. R. Yager, "On ordered weighted averaging aggregation operators in multi-criteria decision making," IEEE Trans. on Systems, Man and Cybernetics, vol. 18, no. 1, (1988), pp. 183-190.

[23] Z. S. Xu and R. R. Yager, "Some geometric aggregation operators based on intuitionistic fuzzy sets," 
International Journal of General Systems, vol. 35, no. 4, (2006), pp. 417-433.

[24] H. Zhao, Z. S. Xu, M. F. Ni and S. S. Liu, "Generalized aggregation operators for intuitionistic fuzzy sets," International Journal of Intelligent Systems, vol. 25, no. 1, (2010), pp. 1-30.

[25] R. R. Yager, “The power average operator”, IEEE Trans. on Systems, Man, Cybernetics-Part A:Systems and Humans, vol. 31, (2001), pp. 724-731.

[26] Z. S. Xu and R. R. Yager, "Power-geometric operators and their use in group decision making," IEEE Trans. on Fuzzy Systems, vol. 18, (2010), pp. 94-105.

[27] L. G. Zhou, H. Y. Chen and J. P. Liu, "Generalized power aggregation operators and their applications in group decision making”, Computers \& Industrial Engineering, vol. 62, (2012), pp. 989-999.

[28] P. D. Liu and Y. Liu, "An approach to multiple attribute group decision making based on intuitionistic trapezoidal fuzzy power generalized aggregation operator", International Journal of Computational Intelligence Systems, vol. 7, no. 2, (2014), pp. 291-304.

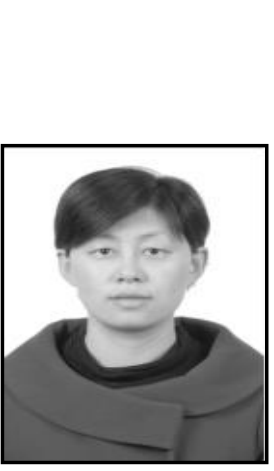

\section{Authors}

Lihua Yang, She is a Lecturer, School of Economics and Management, Hubei University of Automotive Technology. She received her B.S degree from Yangtze University in 2003, and her M.S. degree from Southwest Jiaotong University in 2006.Her research interests include data mining and decision support.

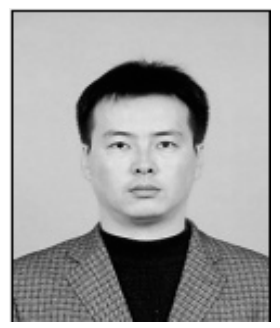

Baolin Li, He is a Lecturer, School of Economics and Management, Hubei University of Automotive Technology. He received his B.S degree from Lanzhou University in 2005, and his M.S. degree from Northwestern Polytechnical University in 2012. His research interests include data analysis and data management. 